\title{
Performance Analysis of Reconfigurable Intelligent Surface-Aided Full-Duplex Cooperative NOMA System
}

\author{
Xiang Zhao $\mathbb{D}^{1}$ and Jinyong Sun $\mathbb{B}^{2}$ \\ ${ }^{1}$ Key Laboratory of Cognitive Radio and Information Processing, Guilin University of Electronic Technology, Guilin 541004, China \\ ${ }^{2}$ Guangxi Key Laboratory of Trusted Software, Guilin University of Electronic Technology, Guilin 541004, China
}

Correspondence should be addressed to Jinyong Sun; sunjy@guet.edu.cn

Received 5 February 2021; Revised 26 June 2021; Accepted 17 July 2021; Published 2 August 2021

Academic Editor: Luis Castedo

Copyright ( 2021 Xiang Zhao and Jinyong Sun. This is an open access article distributed under the Creative Commons Attribution License, which permits unrestricted use, distribution, and reproduction in any medium, provided the original work is properly cited.

\begin{abstract}
The outage performance of a reconfigurable intelligent surface- (RIS-) aided full-duplex cooperative nonorthogonal multiple access (NOMA) system is studied in this paper. Based on the statistical characteristics of the signal-to-noise ratio of the reflection channel from the access point via RIS to the near user, and the cooperative channel from the near user to the far NOMA user, the outage probability of both the near and far users is derived. Through the comparison with the outage performance of conventional cooperative NOMA without employing RIS, the superiority of the proposed scheme is demonstrated. Finally, the correctness of the analytical results is validated with simulation.
\end{abstract}

\section{Introduction}

As a revolutionizing technology for future wireless communications, reconfigurable intelligent surfaces (RISs) have gained tremendous attention recently due to their ability to reconfigure wireless propagation environments $[1,2]$. RISs consist of passive metasurfaces which can change and reflect the direction of the incident signal to an expected destination under a smart controller. RISs can operate in a nearly passive full-duplex mode without resulting in self-interference [3]. RISs can replace radio frequency (RF) chains as a novel modulator, leading RISs-aided wireless systems to be low in cost and energy consumption. To satisfy different requirements, RISs-aided wireless systems have been combined with other advanced technologies, such as an unmanned aerial vehicle (UAV) [4], visible light communication (VLC) [5], physical layer security [6], multiuser communication [7, 8], multiantenna communication [9], and nonorthogonal multiple access (NOMA) $[10,11]$ to enhance system performance.

The integration of RIS into NOMA leads to a novel vision of a network with the features of high cost efficiency, superior spectrum/energy efficiency, and wide area coverage. Great effort has been put into the study of RIS-NOMA. In particu- lar, Ref. [12] investigated the transmit power minimization via joint designing power allocation of the access point (AP) and the phase shifts of RISs. Ref. [13] studied the impact of the random discrete and coherent phase shifts on the reliability of the RIS-NOMA system. Ref. [14] investigated the user pairing for a RIS-aided NOMA system and made a comparison with a series of RIS-assisted orthogonal multiple access systems.

However, little has been focused on the integration of RIS into cooperative NOMA. Cooperative NOMA $[15,16]$, based on prior information available in NOMA and cooperation among users, can further improve system performance and extend communication coverage. Motivated by the advantages of IRS and cooperative NOMA, in this work, we study the outage performance of the RISs-aided full-duplex cooperative NOMA system. Note that prior work [17] has studied the RIS-aided user-cooperation relay NOMA system. However, it was carried out from an optimization perspective and formulated a total transmission power minimization problem subject to the RIS's passive beamforming and the power control of both the AP and the user relay.

In this work, we investigate the outage performance of the RISs-aided full-duplex cooperative NOMA system from 


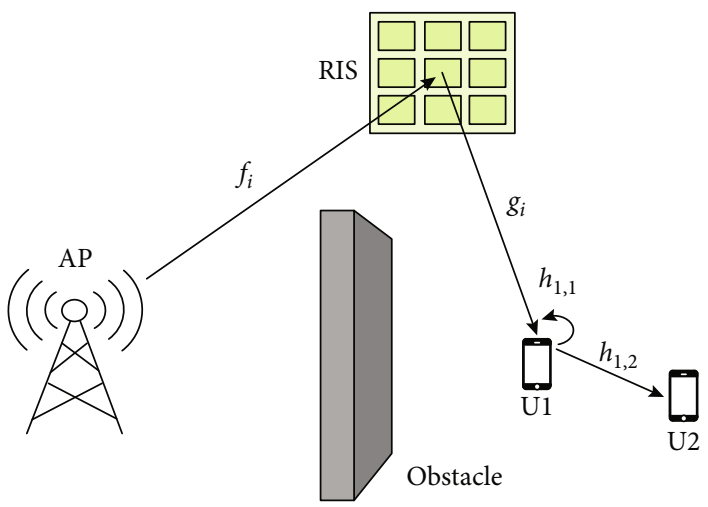

Figure 1: System model of the RIS-aided full-duplex downlink cooperative NOMA communication system.

TABLE 1: System parameters.

\begin{tabular}{|c|c|}
\hline Parameters & Descriptions \\
\hline$f_{i}$ & Gain of the channel from the AP to the $i$ th element of the RIS \\
\hline$g_{i}$ & Gain of the channel from the $i$ th element of the RIS to U1 \\
\hline$\lambda_{i}$ & Reflectance factor of the $i$ th element of the RIS \\
\hline$h_{1}$ & Gain of the reflection channel from the AP via RIS to U1 \\
\hline$h_{1,1}$ & Gain of the residual self-interference channel from U1 to U1 \\
\hline$h_{1,2}$ & Gain of the NOMA cooperative channel from U1 to U2 \\
\hline$l$ & Propagation distance from the AP to the RIS \\
\hline$d$ & Propagation distance from the RIS to U1 \\
\hline$\mu_{i}$ & Amplitude of the Rayleigh fading of $f_{i}$ \\
\hline$v_{i}$ & Amplitude of the Rayleigh fading of $g_{i}$ \\
\hline$\phi_{i}$ & Phase of the Rayleigh fading of $f_{i}$ \\
\hline$\theta_{i}$ & Phase of the Rayleigh fading of $g_{i}$ \\
\hline$\varphi_{i}$ & Reconfigurable phase of the $i$ th element of the RIS \\
\hline$\varepsilon$ & Path-loss index \\
\hline
\end{tabular}

a probability perspective and through the comparison with the outage performance of conventional cooperative NOMA without employing RIS demonstrate the superiority of the proposed system. Finally, the correctness of the analytical results is validated with simulation.

\section{System and Channel Model}

The considered RISs-aided full-duplex downlink cooperative NOMA system model is shown in Figure 1, which includes an AP and a RIS consisting of $N$ elements and two prepaired NOMA users (U1 and U2). The assumption for considering two cooperative NOMA users is the same as [16]. The direct link between the AP and NOMA users does not exist due to obstacle objects or heavy shadowing, and only the reflected signal from the RIS can be received by NOMA users. We call $\mathrm{U} 1$ the near user who is near to the RIS, and U2 the far user. According to the user-relay cooperative NOMA principle, $\mathrm{U} 1$ can obtain the prior information of $\mathrm{U} 2$ via successive interference cancellation (SIC) [18]. Thus, U1 acts as a relay to forward the information to U2. Here, a full-duplex relay is utilized by $\mathrm{U} 1$ performing receiving information and relay transmission simultaneously. The proposed model can be applied to improve the performance of the cell edge users in multicell cellular systems or enhance the reliability of a device-to-device (D2D) communication system. It should be noted that if the direct link is available then more power will be received for both $\mathrm{U} 1$ and $\mathrm{U} 2$; thus, the system performance will be improved given the residual self-interference. Therefore, the performance achieved for the proposed system is a lower limit.

For the proposed RIS-aided full-duplex downlink cooperative NOMA system, the gain $h_{1}$ of the reflection channel from the AP via RIS to U1 can be given as $h_{1}=\sum_{i=1}^{N} f_{i} \lambda_{i} g_{i}$, where $f_{i}$ and $g_{i}$ are, respectively, the gain of the channel from the AP to the $i$ th $(i=1, \cdots, N)$ element of the RIS and from the $i$ th element of the RIS to U1, given by $f_{i}=l^{-\varepsilon / 2} \mu_{i} \exp (-$ $\left.j \phi_{i}\right)$ and $g_{i}=d^{-\varepsilon / 2} v_{i} \exp \left(-j \theta_{i}\right)$. The $l$ and $d$ are the propagation distances from the AP to the RIS and from the RIS to U1, respectively. The $\varepsilon$ is the path-loss index, being a constant in 


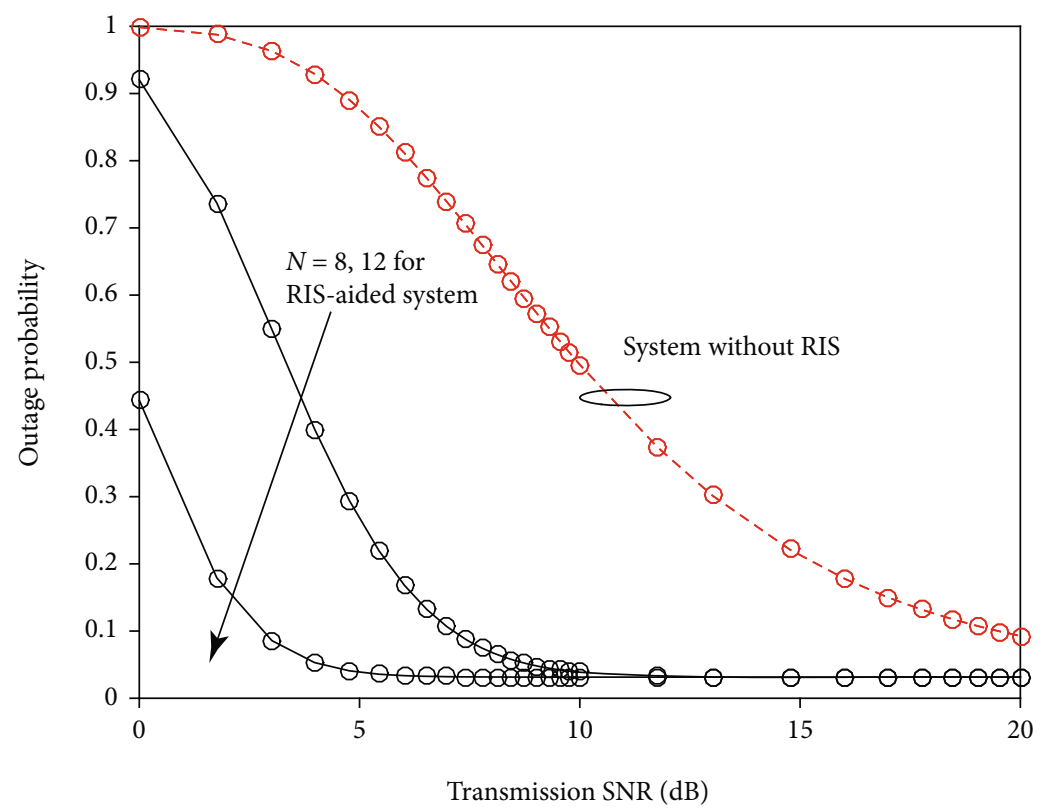

(a)

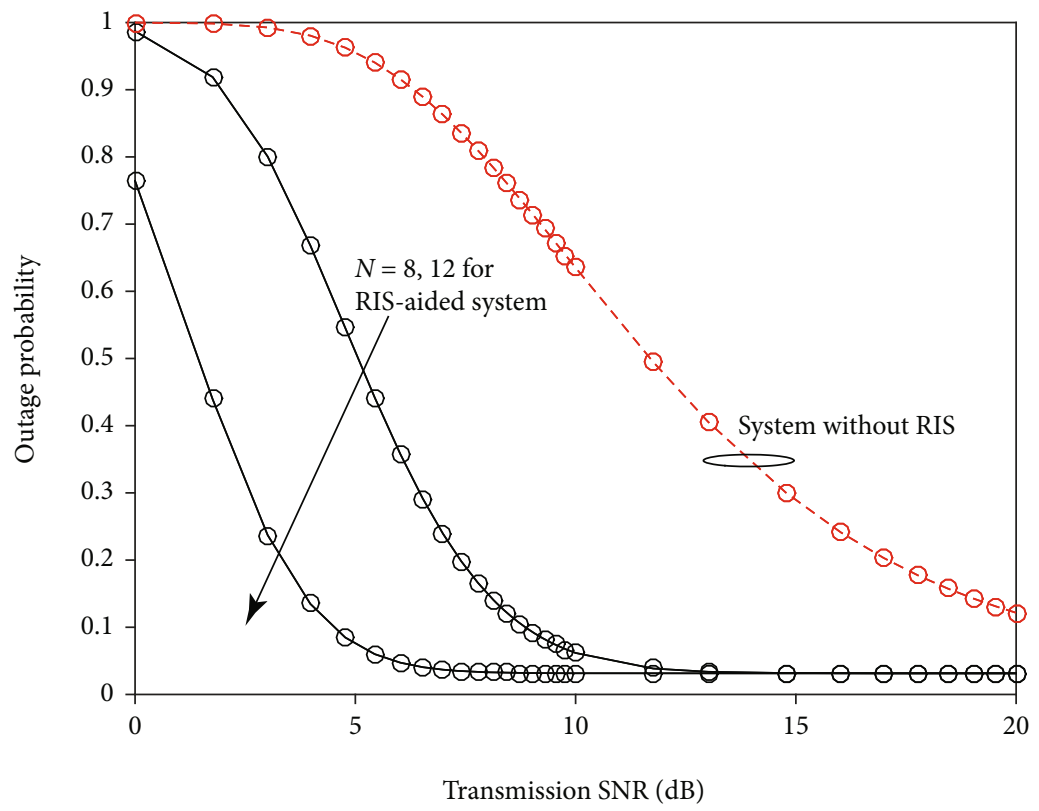

(b)

Figure 2: Continued. 


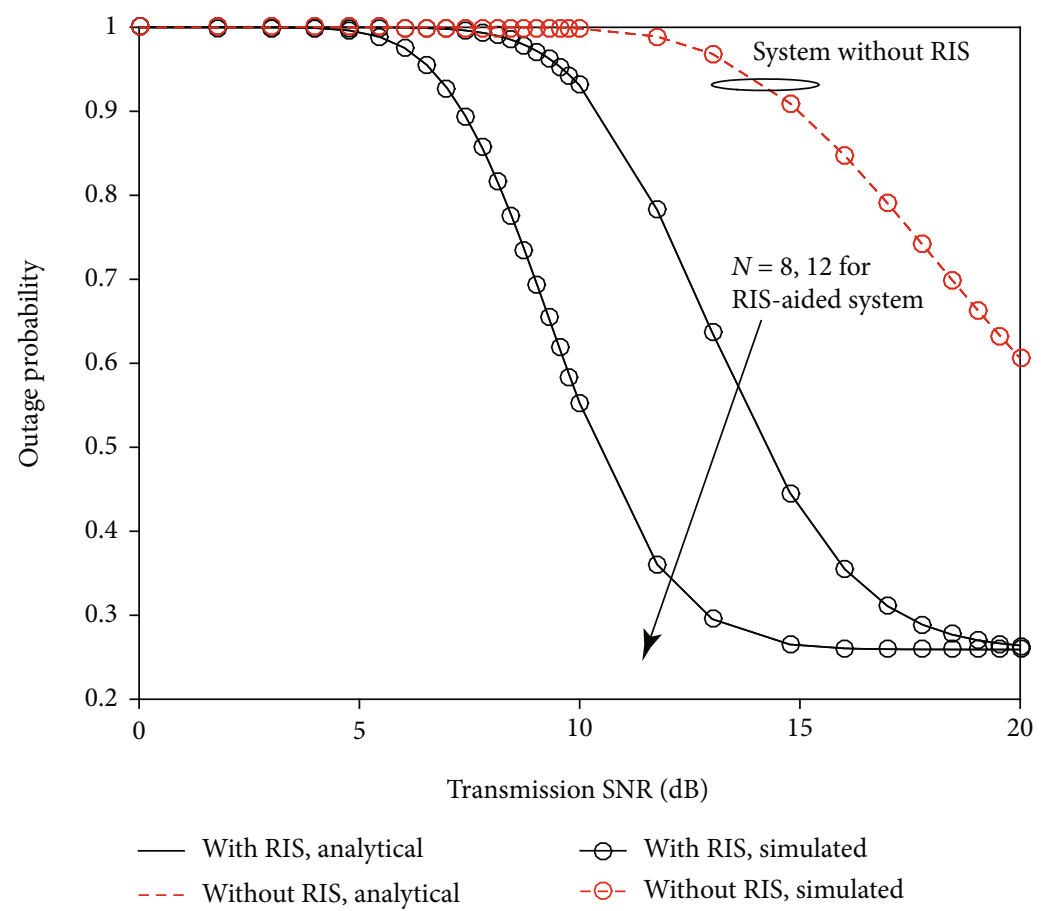

(c)

FIGURE 2: The far user's outage probability comparison between the RIS-aided full-duplex cooperative NOMA system and the cooperative NOMA system without RIS for various values of $N$ under $\gamma_{S}=0 \mathrm{~dB}$ : (a) $w_{1}=0.1, R_{\mathrm{th}}=0.2 \mathrm{~b} / \mathrm{s} / \mathrm{Hz}$; (b) $w_{1}=0.4, R_{\mathrm{th}}=0.2 \mathrm{~b} / \mathrm{s} / \mathrm{Hz}$; (c) $w_{1}=$ $0.1, R_{\mathrm{th}}=1 \mathrm{~b} / \mathrm{s} / \mathrm{Hz}$.

this paper. $\mu_{i}$ and $\phi_{i}$ represent the amplitude and phase of $f_{i}$, respectively, and $v_{i}$ and $\theta_{i}$ denote the amplitude and phase of $g_{i}$, respectively. $\mu_{i}$ and $\nu_{i}$ are assumed to follow Rayleigh distribution, and $\phi_{i}$ and $\theta_{i}$ can be estimated. How to get $\phi_{i}$ and $\theta_{i}$ is beyond the scope of this paper and can be referenced from [2]. The reflectance factor of the $i$ th $(i=1, \cdots, N)$ element of the RIS is $\lambda_{i}=\exp \left(j \varphi_{i}\right)$, where the reconfigurable phase $\varphi_{i}$ of the RIS can be deployed as $\varphi_{i}=\phi_{i}+\theta_{i}$ in order to make the signal-to-noise ratio (SNR) of the near user U1 maximum [3]. The system parameters employed in this work are included in Table 1. fied as

Hence, the gain $h_{1}$ of the reflection channel can be simpli-

$$
h_{1}=(l d)^{-\varepsilon / 2} \sum_{i=1}^{N} \mu_{i} v_{i}
$$

For the RIS-aided full-duplex cooperative NOMA system, the near user U1 performs SIC and forwards the detected information to the far user $\mathrm{U} 2$ at the time of receiving the signal from RIS; meanwhile, U1 suffers residual selfinterference. Thus, the received signal $y_{1}$ by $\mathrm{U} 1$ can be written as

$$
y_{1}=h_{1} \sqrt{P_{s}}\left(w_{1} s_{1}+w_{2} s_{2}\right)+h_{1,1} \sqrt{P_{1}} x+n_{1} \text {, }
$$

where $P_{s}$ is the transmit power of the AP; the information signal $s_{m}$ intended for the $m$ th NOMA user, $m \in\{1,2\}$, is allocated power with a coefficient of $w_{m}$ that satisfies $w_{1}<$ $w_{2}$ and $w_{1}^{2}+w_{2}^{2}=1$. The gain of the residual selfinterference channel from U1 to U1 is $h_{1,1}$. U1 transmits with power $P_{1}$, and the self-interference signal $x$ satisfies $E\left[|x|^{2}\right]$ $=1$, where $E(\cdot)$ is the expectation operation. $n_{1}$ is the additive white Gaussian noise (AWGN) with zero mean and variance $N_{1}$.

The received signal $y_{2}$ by $\mathrm{U} 2$ is given by

$$
y_{2}=h_{1,2} \sqrt{P_{1}} s_{2}+n_{2}
$$

where $h_{1,2}$ is the gain of the NOMA cooperative channel from $\mathrm{U} 1$ to U2. $n_{2}$ is the AWGN of this channel with zero mean and variance $N_{2}$.

Employing SIC technology, the near user U1 first decodes the information of the far user $\mathrm{U} 2$ and then subtracts it from the received signal in order to recover its own information. Thus, the signal-to-interference-plus-noise ratio (SINR) at $\mathrm{U} 1$ to detect information $s_{2}$ of $\mathrm{U} 2$ can be expressed as $Q_{1,2}$ $=P_{s} w_{2}^{2}\left|h_{1}\right|^{2} /\left(P_{s} w_{1}^{2}\left|h_{1}\right|^{2}+P_{1}\left|h_{1,1}\right|^{2}+N_{1}\right)$. Let the SNR of the reflection channel from the AP via RIS to U1 be $\gamma_{1}=P_{s}$ $\left|h_{1}\right|^{2} / N_{1}$, and the SNR of the residual self-interference channel from U1 to U1 be $\gamma_{S}=P_{1}\left|h_{1,1}\right|^{2} / N_{1}$, then the received SINR at U1 to detect information $s_{2}$ of U2 can be finally expressed as

$$
Q_{1,2}=\frac{w_{2}^{2} \gamma_{1}}{w_{1}^{2} \gamma_{1}+\gamma_{S}+1}
$$




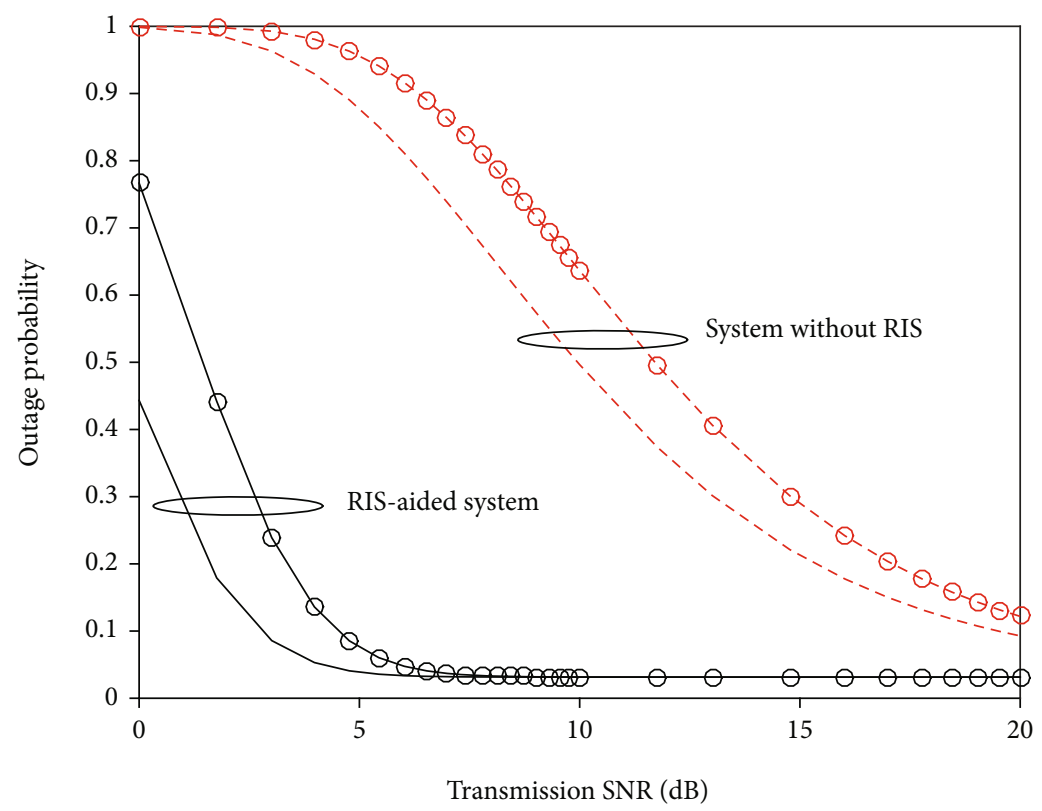

(a)

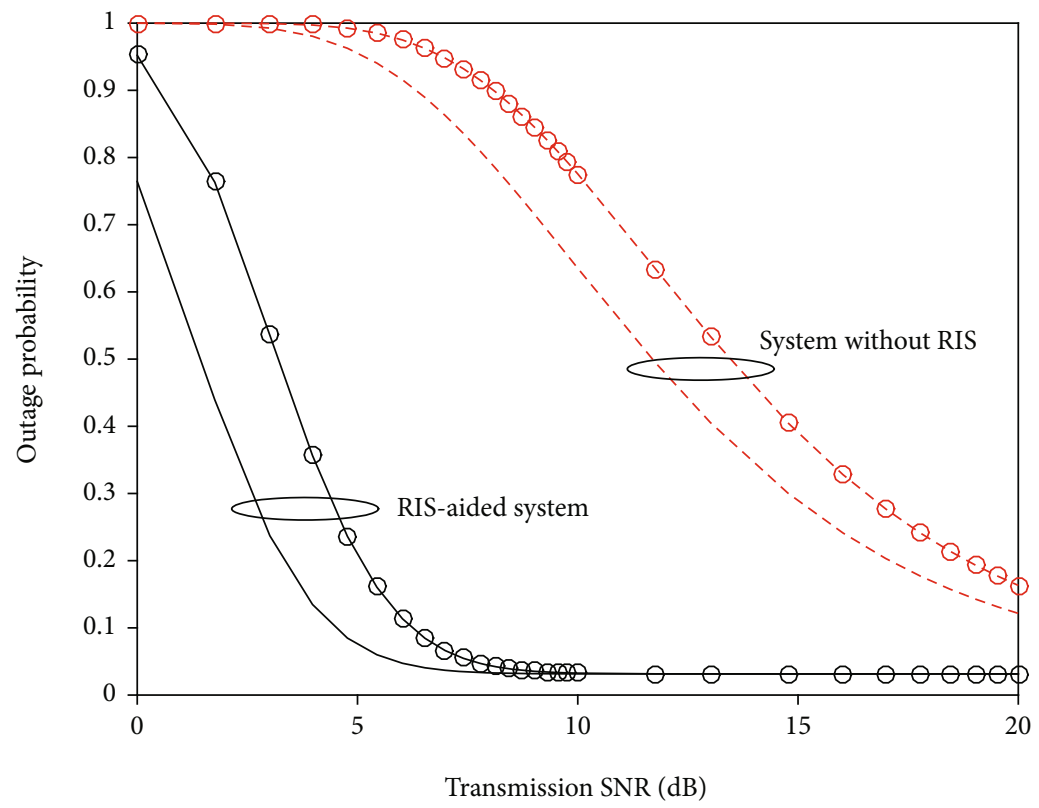

(b)

Figure 3: Continued. 


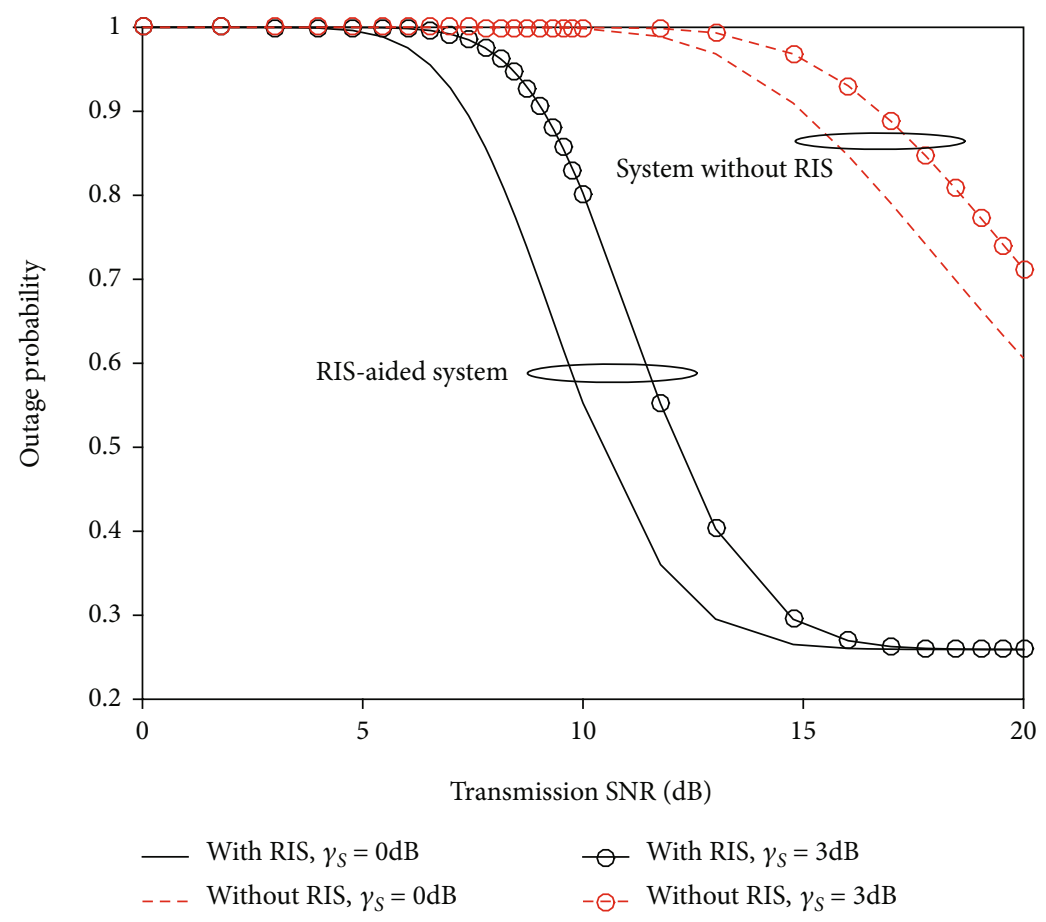

(c)

FIGURE 3: The far user's outage probability comparison between the RIS-aided cooperative NOMA system and the cooperative NOMA system without RIS for various values of residual self-interference $\gamma_{S}$ under $N=12$ : (a) $w_{1}=0.1, R_{\mathrm{th}}=0.2 \mathrm{~b} / \mathrm{s} / \mathrm{Hz} ;$ (b) $w_{1}=0.4, R_{\mathrm{th}}=0.2 \mathrm{~b} / \mathrm{s} / \mathrm{Hz}$; (c) $w_{1}=0.1, R_{\mathrm{th}}=1 \mathrm{~b} / \mathrm{s} / \mathrm{Hz}$.

The received SINR at $\mathrm{U} 1$ to detect its own information $s_{1}$ can be expressed as

$$
Q_{1,1}=\frac{P_{s} w_{1}^{2}\left|h_{1}\right|^{2}}{P_{1}\left|h_{1,1}\right|^{2}+N_{1}}=\frac{w_{1}^{2} \gamma_{1}}{\gamma_{S}+1}
$$

On condition that $\mathrm{U} 1$ can detect information $s_{2}$ of $\mathrm{U} 2$, the received SNR at $\mathrm{U} 2$ to detect its own information $s_{2}$ can be given by

$$
\gamma_{2}=\frac{P_{1}\left|h_{1,2}\right|^{2}}{N_{2}}
$$

\section{Statistical Characteristics of SNR}

In order to get the performance of the RIS-aided full-duplex cooperative NOMA system, it is necessary to determine the statistical characteristics of $\gamma_{1}, \gamma_{2}$, and $\gamma_{S}$.

For the SNR of the reflection channel from the AP via RIS to U1, $\gamma_{1}=\bar{\gamma}_{1} X^{2}$, where $\bar{\gamma}_{1}$ is the average SNR given by $\bar{\gamma}_{1}$ $=P_{s}(l d)^{-\varepsilon} / N_{1}$, and $X=\sum_{i=1}^{N} X_{i}=\sum_{i=1}^{N} \mu_{i} v_{i}$. From [19], we know that if channel amplitude $\mu_{i}$ and $\nu_{i}$ is an independently identically Rayleigh distributed random variable with mean $\sqrt{\pi} / 2$ and variance $(1-\pi / 4)$, then $X^{2}$ obeys squared $K_{G}$ distribution, and the probability density function (PDF) of $\gamma_{1}$ can be written as

$$
f_{\gamma_{1}}(r)=\frac{2 \Lambda^{a+b}}{\Gamma(a) \Gamma(b)} r^{(a+b) / 2-1} K_{a-b}(2 \Lambda \sqrt{r})
$$

and the cumulative distribution function (CDF) of $\gamma_{1}$ can be given by

$$
F_{\gamma_{1}}(r)=\int_{0}^{r} f_{\gamma_{1}}(u) d u=\frac{1}{\Gamma(a) \Gamma(b)} G_{1,3}^{2,1}\left[\left.\Lambda^{2} r\right|_{a, b, 0} ^{1}\right],
$$

where $G_{q_{1}, q_{2}}^{q_{3}, q_{4}}(\cdot)$ is Meijer's $G$-function [20] (Eq.(9.301)) with $q_{1}, q_{2}, q_{3}, q_{4}$ being the parameters; $K_{n}(\cdot)$ is the modified $n$ th order Bessel function of the second kind [20] (Eq.(8.432)); $\Gamma(\cdot)$ is the gamma function [20] (Eq.(8.310.1)); $\Lambda$ has relation to both the $K_{G}$ distribution parameters and the 2 nd order moment of $X$, which is expressed by $\Lambda=$ $\sqrt{a b / \bar{\gamma}_{1} \beta_{X}(2)}$, where $a$ and $b$ are the $K_{G}$ distribution parameters and $\beta_{X}(2)$ is the 2 nd order moment of $X$. Each of $X^{\prime}$ 's $j$ th order moment is given by

$\beta_{X}(j)=\sum_{j_{1}=0}^{j} \sum_{j_{2}=0}^{j_{1}} \cdots \sum_{j_{N-1}=0}^{j_{N-2}}\left(\begin{array}{l}j \\ j_{1}\end{array}\right)\left(\begin{array}{l}j_{1} \\ j_{2}\end{array}\right) \cdots\left(\begin{array}{c}j_{N-2} \\ j_{N-1}\end{array}\right) \cdot \beta_{X_{i}}\left(j-j_{1}\right) \beta_{X_{i}}\left(j_{1}-j_{2}\right) \cdots \beta_{X_{i}}\left(j_{N-1}\right)$,

where $\beta_{X_{i}}(j)=[\Gamma(1+j / 2)]^{2}$. With the 2 nd order, 4 th order, and 6 th order moments of $X$, we can finally get $a$ and $b$ by utilizing Eqs. (14)-(18) of [21]. 


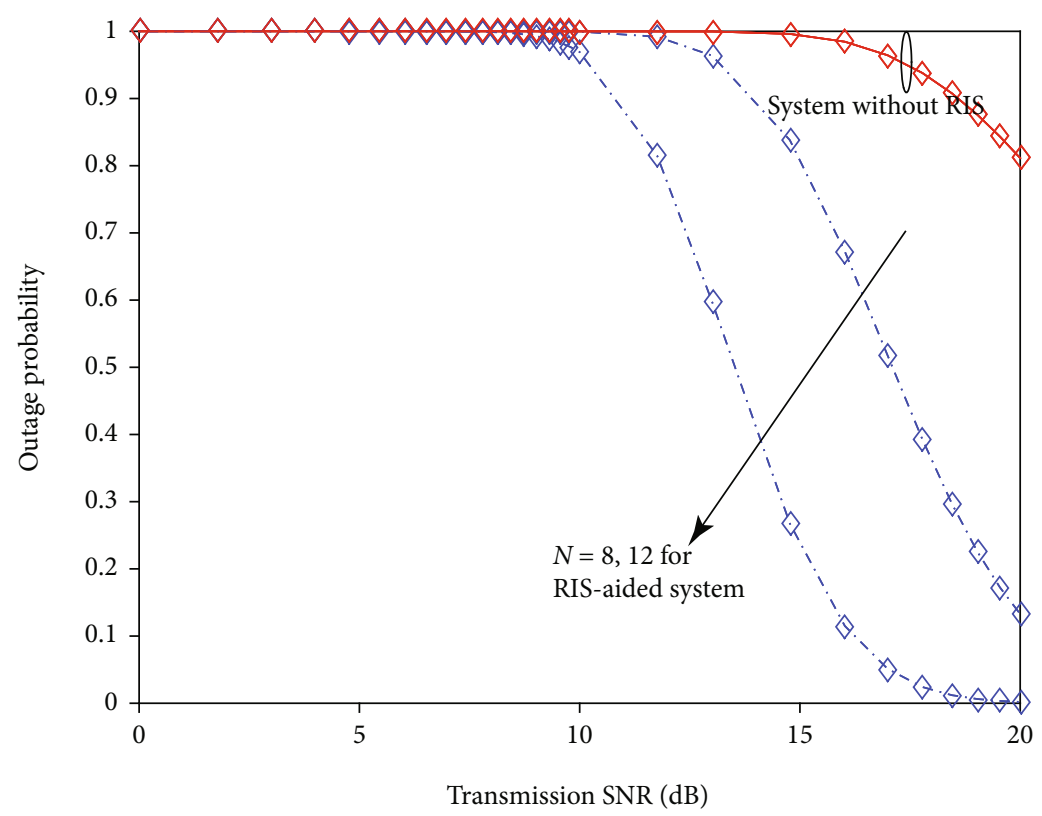

(a)

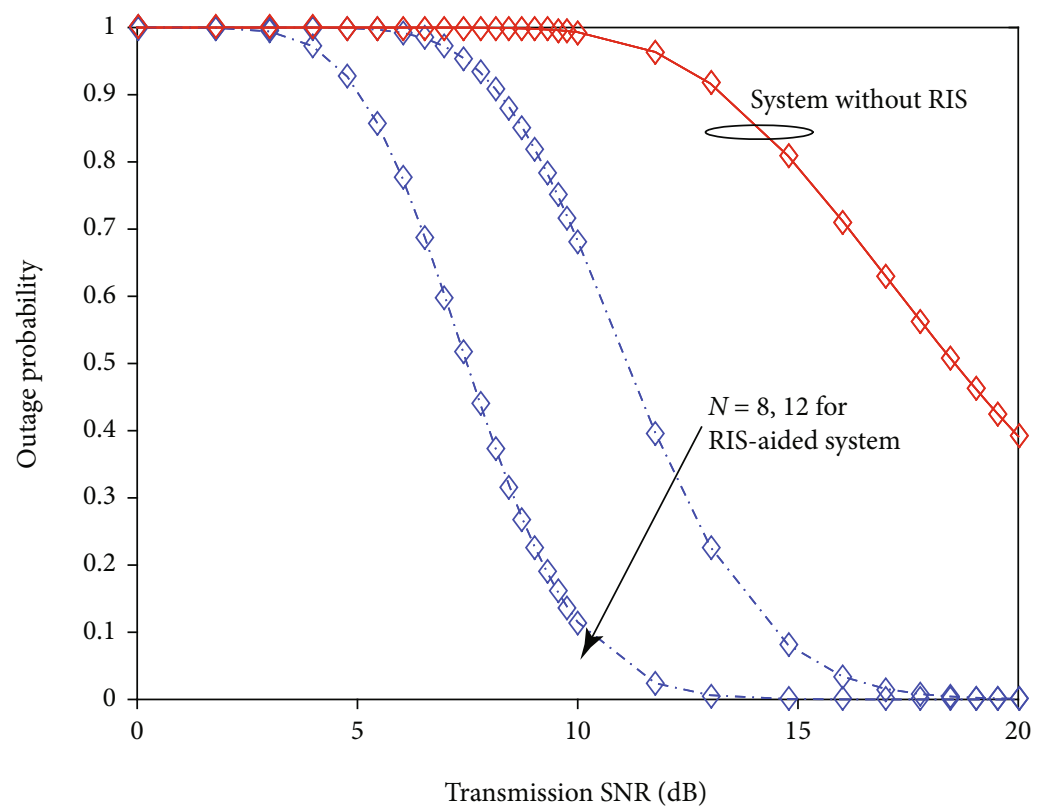

(b)

Figure 4: Continued. 


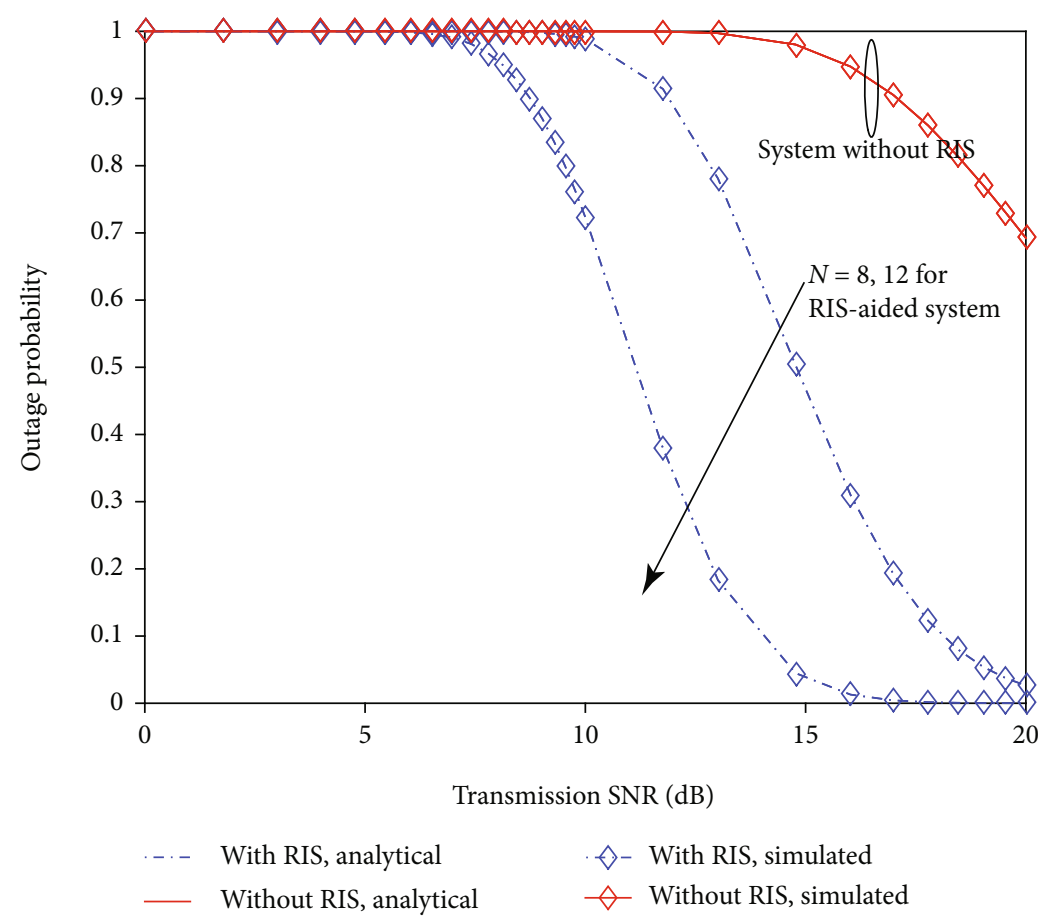

(c)

FIGURE 4: The near user's outage probability comparison between the RIS-aided full-duplex cooperative NOMA system and the cooperative NOMA system without RIS for various values of $N$ under $\gamma_{S}=0 \mathrm{~dB}$ : (a) $w_{1}=0.2, R_{\mathrm{th}}=0.2 \mathrm{~b} / \mathrm{s} / \mathrm{Hz}$; (b) $w_{1}=0.4, R_{\mathrm{th}}=0.2 \mathrm{~b} / \mathrm{s} / \mathrm{Hz}$; (c) $w_{1}=0.4$, $R_{\mathrm{th}}=0.4 \mathrm{~b} / \mathrm{s} / \mathrm{Hz}$.

For the cooperative NOMA channel from U1 to U2, considering that $h_{1,2}$ follows Rayleigh fading, $\gamma_{2} \sim \operatorname{Exp}\left(1 / \bar{\gamma}_{2}\right)$ always holds, where $\bar{\gamma}_{2}=\mathrm{E}\left[\left|h_{1,2}\right|^{2}\right] P_{1} / N_{2}$.

For simplicity, the SNR $\gamma_{S}$ of the residual self-interference channel from $\mathrm{U} 1$ to $\mathrm{U} 1$ is a constant in this paper.

\section{Performance Analysis}

When the achievable data rate of a wireless link is below the target rate, an outage occurs. In the following, we first obtain the outage probability (OP) of the RIS-aided full-duplex cooperative NOMA system; for the comparison, we also get the OP of the cooperative NOMA system without employing RIS.

4.1. OP of the RIS-Aided Full-Duplex Cooperative NOMA System. Outage experienced by the near user U1 follows two situations. One is $\mathrm{U} 1$ cannot detect $s_{2}$ of $\mathrm{U} 2$ resulting in the break of SIC. The other is U1 can detect $s_{2}$ of U2 but U1's overall received SINR cannot support the target rate. Therefore, the OP of the near user U1 can be written as

$$
\begin{aligned}
P_{1, \text { out }}= & \operatorname{Pr}\left(\frac{1}{2} \log \left(1+Q_{1,2}\right)<R_{\mathrm{th} 0}\right) \\
& +\operatorname{Pr}\left(\frac{1}{2} \log \left(1+Q_{1,2}\right)>R_{\mathrm{th} 0}, \frac{1}{2} \log \left(1+Q_{1,1}\right)<R_{\mathrm{th} 1}\right),
\end{aligned}
$$

where the target rate $R_{\text {tho }}$ is to limit the data rate achieved by the reflection channel from the AP via RIS to U1 for decoding the information of $\mathrm{U} 2$, and the target rate $R_{\mathrm{th} 1}$ is employed by $\mathrm{U} 1$ to detect its own information.

Similarly, the outage experienced by the far user U2 also follows two situations. One is U1 cannot detect $s_{2}$ of U2 causing $\mathrm{U} 2$ not receiving information at all. The other is $\mathrm{U} 1 \mathrm{can}$ detect and forward $s_{2}$ of U2 but U2's overall received SINR cannot support the target rate $R_{\text {th2 }}$. Therefore, the OP of the far user $\mathrm{U} 2$ can be written as

$$
\begin{aligned}
P_{2, \text { out }}= & \operatorname{Pr}\left(\frac{1}{2} \log \left(1+Q_{1,2}\right)<R_{\mathrm{th} 0}\right) \\
& +\operatorname{Pr}\left(\frac{1}{2} \log \left(1+Q_{1,2}\right)>R_{\mathrm{th} 0}, \frac{1}{2} \log \left(1+Q_{2}\right)<R_{\mathrm{th} 2}\right) .
\end{aligned}
$$

In the following, the OP of the near user U1 and far user $\mathrm{U} 2$ will be calculated, respectively.

Let $\tau_{0}=2^{2 R_{\mathrm{th} 0}}-1, \tau_{1}=2^{2 R_{\mathrm{th} 1}}-1, \tau_{2}=2^{2 R_{\mathrm{th} 2}}-1$. The OP of U1 for the RIS-aided full-duplex cooperative NOMA system can be written as

$$
\begin{aligned}
P_{1, \text { out }} & =\operatorname{Pr}\left(Q_{1,2}<\tau_{0}\right)+\operatorname{Pr}\left(Q_{1,2}>\tau_{0}, Q_{1,1}<\tau_{1}\right) \\
& =\operatorname{Pr}\left(Q_{1,2}<\tau_{0}\right)+\operatorname{Pr}\left(Q_{1,2}>\tau_{0}\right) \operatorname{Pr}\left(Q_{1,1}<\tau_{1}\right) \\
& =\operatorname{Pr}\left(Q_{1,2}<\tau_{0}\right)+\left[1-\operatorname{Pr}\left(Q_{1,2}<\tau_{0}\right)\right] \operatorname{Pr}\left(Q_{1,1}<\tau_{1}\right) .
\end{aligned}
$$

Obviously, it is necessary to first determine $\operatorname{Pr}\left(Q_{1,2}<\tau_{0}\right)$ 


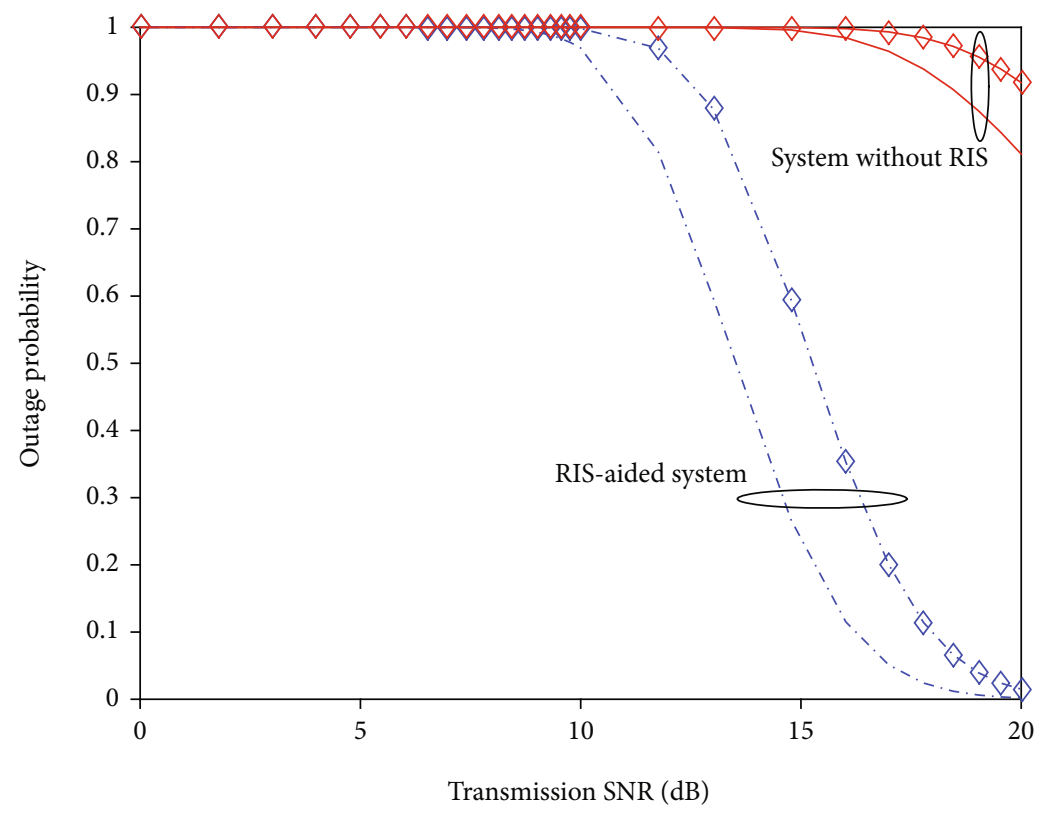

(a)

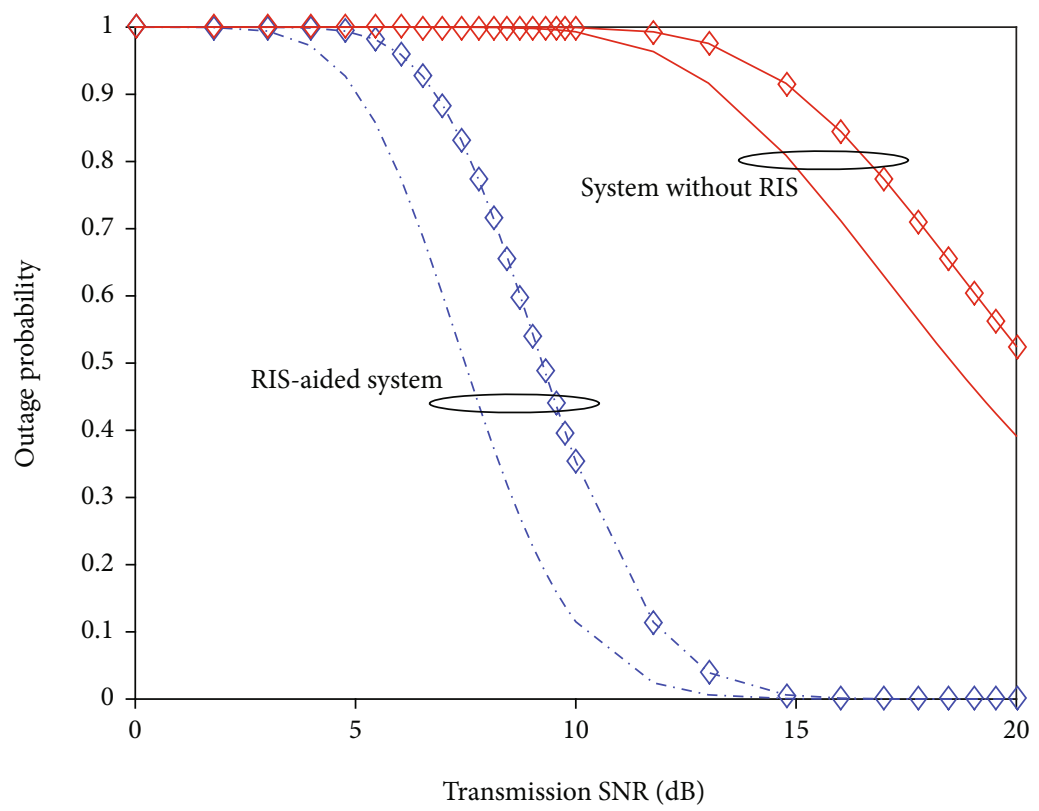

(b)

Figure 5: Continued. 


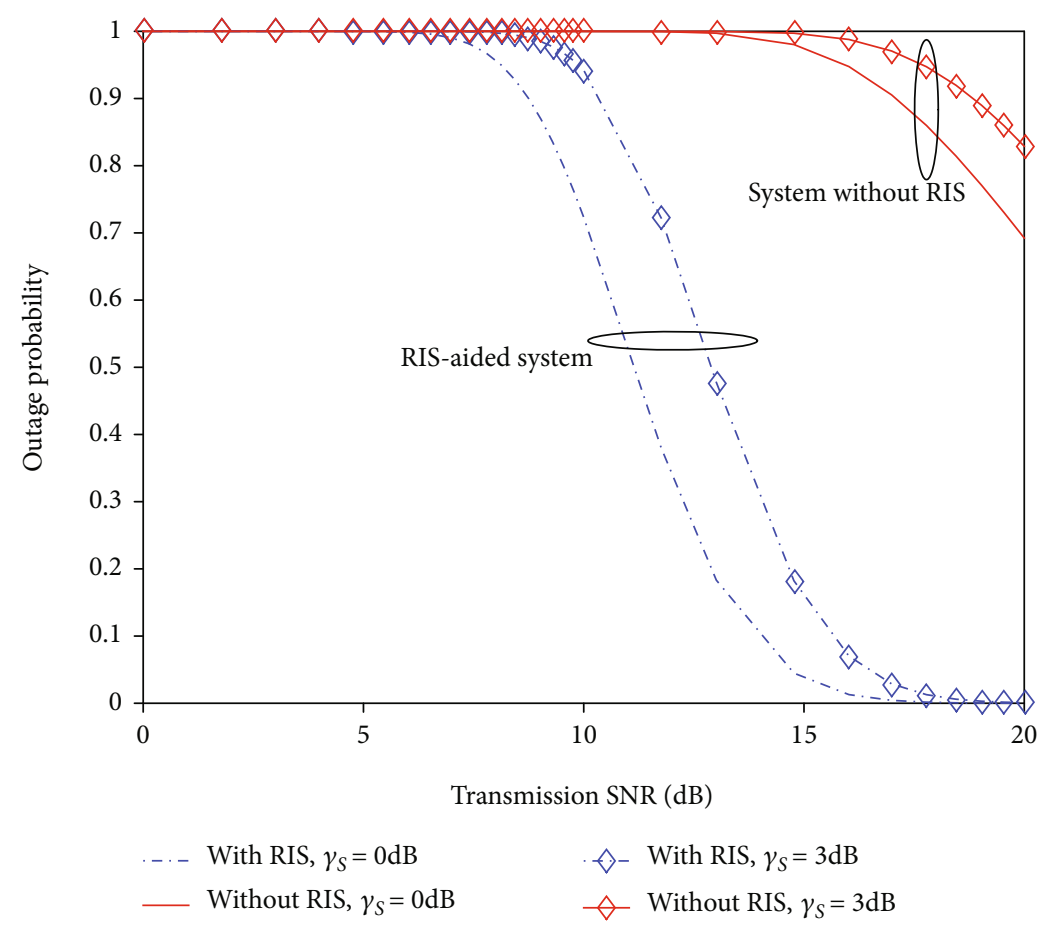

(c)

FIGURE 5: The near user's outage probability comparison between the RIS-aided cooperative NOMA system and the cooperative NOMA system without RIS for various values of residual self-interference $\gamma_{S}$ under $N=12$ : (a) $w_{1}=0.2, R_{\mathrm{th}}=0.2 \mathrm{~b} / \mathrm{s} / \mathrm{Hz}$; (b) $w_{1}=0.4, R_{\mathrm{th}}=0.2$ $\mathrm{b} / \mathrm{s} / \mathrm{Hz} ;(\mathrm{c}) w_{1}=0.4, R_{\mathrm{th}}=0.4 \mathrm{~b} / \mathrm{s} / \mathrm{Hz}$.

and $\operatorname{Pr}\left(Q_{1,1}<\tau_{1}\right)$ before obtaining $P_{1, \text { out }}$. Since $\lim _{\gamma_{1} \longrightarrow \infty} Q_{1,2}$ $=\lim _{\gamma_{1} \longrightarrow \infty} w_{2}^{2} \gamma_{1} /\left(w_{1}^{2} \gamma_{1}+\gamma_{s}+1\right)=w_{2}^{2} / w_{1}^{2}$, if $\tau_{0} \geq w_{2}^{2} / w_{1}^{2}$, then $\operatorname{Pr}\left(Q_{1,2}<\tau_{0}\right)=1$ holds. If $0<\tau_{0}<w_{2}^{2} / w_{1}^{2}$, then $\operatorname{Pr}\left(Q_{1,2}<\tau_{0}\right.$ )$=\operatorname{Pr}\left(\gamma_{1}<\tau_{0}\left(\gamma_{s}+1\right) /\left(w_{2}^{2}-\tau_{0} w_{1}^{2}\right)\right)=F_{\gamma_{1}}\left(\tau_{0}\left(\gamma_{s}+1\right) /\left(w_{2}^{2}-\right.\right.$ $\left.\left.\tau_{0} w_{1}^{2}\right)\right)$ can be obtained, where $w_{2}^{2}>\tau_{0} w_{1}^{2}$ should be always satisfied according to the NOMA protocol. In brief, $\operatorname{Pr}\left(Q_{1,2}\right.$ $<\tau_{0}$ ) can be given by

$$
\operatorname{Pr}\left(Q_{1,2}<\tau_{0}\right)= \begin{cases}1, & \tau_{0} \geq \frac{w_{2}^{2}}{w_{1}^{2}} \\ F_{\gamma_{1}}\left(\frac{\tau_{0}\left(\gamma_{s}+1\right)}{w_{2}^{2}-\tau_{0} w_{1}^{2}}\right), & 0<\tau_{0}<\frac{w_{2}^{2}}{w_{1}^{2}} .\end{cases}
$$

Similarly, $\operatorname{Pr}\left(Q_{1,1}<\tau_{1}\right)$ can be given by

$\operatorname{Pr}\left(Q_{1,1}<\tau_{1}\right)=\operatorname{Pr}\left(\gamma_{1}<\frac{\tau_{1}\left(\gamma_{S}+1\right)}{w_{1}^{2}}\right)=F_{\gamma_{1}}\left(\frac{\tau_{1}\left(\gamma_{S}+1\right)}{w_{1}^{2}}\right)$.

Hence, the OP of U1 for the RIS-aided full-duplex coop- erative NOMA system can be written as

$$
P_{1, \text { out }}= \begin{cases}1, & \tau_{0} \geq \frac{w_{2}^{2}}{w_{1}^{2}}, \\ F_{\gamma_{1}}\left(\frac{\tau_{0}\left(\gamma_{S}+1\right)}{w_{2}^{2}-\tau_{0} w_{1}^{2}}\right)+\left[1-F_{\gamma_{1}}\left(\frac{\tau_{0}\left(\gamma_{S}+1\right)}{w_{2}^{2}-\tau_{0} w_{1}^{2}}\right)\right] F_{\gamma_{1}}\left(\frac{\tau_{1}\left(\gamma_{S}+1\right)}{w_{1}^{2}}\right), & 0<\tau_{0}<\frac{w_{2}^{2}}{w_{1}^{2}} .\end{cases}
$$

Since $\operatorname{Pr}\left(Q_{2}<\tau_{2}\right)=\int_{0}^{\tau_{2}}\left(1 / \bar{\gamma}_{2}\right) e^{-\left(u / \bar{\gamma}_{2}\right)} d u=1-e^{-\tau_{2} / \bar{\gamma}_{2}}$, the OP of U2 for the RIS-aided full-duplex cooperative NOMA system can be given by

$$
P_{2, \text { out }}= \begin{cases}1, & \tau_{0} \geq \frac{w_{2}^{2}}{w_{1}^{2}} \\ F_{\gamma_{1}}\left(\frac{\tau_{0}\left(\gamma_{S}+1\right)}{w_{2}^{2}-\tau_{0} w_{1}^{2}}\right)+\left[1-F_{\gamma_{1}}\left(\frac{\tau_{0}\left(\gamma_{S}+1\right)}{w_{2}^{2}-\tau_{0} w_{1}^{2}}\right)\right]\left[1-\exp \left(-\frac{\tau_{2}}{\bar{\gamma}_{2}}\right)\right], & 0<\tau_{0}<\frac{w_{2}^{2}}{w_{1}^{2}} .\end{cases}
$$

4.2. OP of the Cooperative NOMA System without RIS. For the comparison, we derive the OP of the cooperative NOMA system without employing RIS, where the AP directly transmit the superimposed signal to $\mathrm{U} 1$, and at the time of receiving the signal, U1 performs SIC and forwards to U2; meanwhile, U1 suffers residual self-interference. Note that there is no link from the AP to U2. The SNR of the channel from the AP to U1 is $\gamma_{1}^{\prime}=P_{s}\left|h_{1}^{\prime}\right|^{2} / N_{1}$, where $h_{1}^{\prime}$ is the gain of the channel from the AP to U1. Considering that this 
channel follows Rayleigh distribution, $\gamma^{\prime}{ }_{1} \sim \operatorname{Exp}\left(1 /{\overline{\gamma^{\prime}}}_{1}\right)$ holds and $\overline{\gamma^{\prime}}$ is the average SNR which can be expressed with $\bar{\gamma}_{1}^{\prime}$
$=E\left[\left|h_{1}^{\prime}\right|^{2}\right] P_{s} / N_{1}$. Thus, for the cooperative NOMA system without RIS, the OP of $\mathrm{U} 1$ is given by

$$
P_{1, \text { out }}^{\prime}= \begin{cases}1, & \tau_{0} \geq \frac{w_{2}^{2}}{w_{1}^{2},} \\ \exp \left(-\frac{\tau_{0}\left(\gamma_{S}+1\right)}{\left(w_{2}^{2}-\tau_{0} w_{1}^{2}\right) \overline{\gamma_{1}^{\prime}}}\right)\left[1-\exp \left(-\frac{\tau_{1}\left(\gamma_{S}+1\right)}{w_{1}^{2} \overline{\gamma_{1}^{\prime}}}\right)\right]+1-\exp \left(-\frac{\tau_{0}\left(\gamma_{S}+1\right)}{\left(w_{2}^{2}-\tau_{0} w_{1}^{2}\right) \overline{\gamma_{1}^{\prime}}}\right), & 0<\tau_{0}<\frac{w_{2}^{2}}{w_{1}^{2}},\end{cases}
$$

and the $\mathrm{OP}$ of $\mathrm{U} 2$ is given by

$$
P_{2, \text { out }}^{\prime}= \begin{cases}1, & \tau_{0} \geq \frac{w_{2}^{2}}{w_{1}^{2}} \\ \exp \left(-\frac{\tau_{0}\left(\gamma_{S}+1\right)}{\left(w_{2}^{2}-\tau_{0} w_{1}^{2}\right) \overline{\gamma_{1}^{\prime}}}\right)\left[1-\exp \left(-\frac{\tau_{2}}{\bar{\gamma}_{2}}\right)\right]+1-\exp \left(-\frac{\tau_{0}\left(\gamma_{S}+1\right)}{\left(w_{2}^{2}-\tau_{0} w_{1}^{2}\right) \overline{\gamma_{1}^{\prime}}}\right), & 0<\tau_{0}<\frac{w_{2}^{2}}{w_{1}^{2}}\end{cases}
$$

\section{Numerical Results and Discussions}

The numerical results for the OP of the RIS-aided full-duplex cooperative NOMA system are presented in this section for a range of parameter values, including the element number $N$ of the metasurface, the NOMA power allocation coefficient $w_{1}$, residual self-interference $\gamma_{S}$, the target rate $R_{\text {th0 }}$ supported by the channel from the AP via RIS to near user U1 for decoding the information of the far user U2, and the target rate $R_{\mathrm{th} 1}$ and $R_{\mathrm{th} 2}$ at which information can be detected by $\mathrm{U} 1$ and $\mathrm{U} 2$, respectively. To investigate the RIS benefits, we compare the outage performance of the RIS-aided fullduplex cooperative NOMA system with that of the cooperative NOMA system without employing RIS. Finally, the Monte-Carlo simulation is employed to verify all the numerical results.

We consider a given user pair (U1, U2) in cooperative NOMA transmission during the simulation process, and the propagation distance from the AP to the RIS is $l=20 \mathrm{~m}$, and the propagation distance from the RIS to U1 is $d=6 \mathrm{~m}$. The transmission SNR $P_{s} / N_{1}$ varies between $0 \mathrm{~dB}$ and $20 \mathrm{~dB}$. For simplicity, we set $R_{\mathrm{th} 0}=R_{\mathrm{th} 1}=R_{\mathrm{th} 2}=R_{\mathrm{th}}$.

The OP of the far user U2 in the RIS-aided full-duplex cooperative NOMA system and the cooperative NOMA system without employing RIS are shown in Figure 2 for various values of the element number $N$ under $\gamma_{S}=0 \mathrm{~dB}$. We can know that no matter how the power allocation coefficient and target rate are, the OP performance of U2 in the RISaided cooperative NOMA system is always superior to that of the cooperative NOMA system without deploying RIS, which demonstrates that precisely controlling the propagation of the signal through the RIS can greatly improve the system performance. We can know from Figure 2 that the simulation results are always consistent with the analytical results. As far as the RIS-aided cooperative NOMA system, we change $N$ from 8 to 12 . The OP performance improves with increasing $N$. For a certain $N$, increasing the transmission SNR can make the OP performance improved. Comparing Figure 2(a) with Figure 2(c), if given the power allocation coefficient to U1, i.e., $w_{1}=0.1$, increasing $R_{\mathrm{th}}$ from $0.2 \mathrm{~b} / \mathrm{s} / \mathrm{Hz}$ to $1 \mathrm{~b} / \mathrm{s} / \mathrm{Hz}$ will make the OP of the far user increase. This means increasing the outage threshold will make the OP performance degraded. Comparing Figure 2(a) with Figure 2(b), we know if given $R_{\text {th }}$ and transmission SNR, the OP performance of the far user gets deteriorated by increasing $w_{1}$. This conclusion agrees with the results of [16], and the reason is that increasing the power allocation efficient $w_{1}$ of the near user will make lower power allocated to the far user and further causing the received SNR of the far user decreased. As shown in Figure 2, if given the outage performance and the noise variance $N_{1}$, the transmission power needed by the cooperative NOMA system without RIS is higher than that with RIS, which is consistent with the results of $[14,17]$.

The OP of the far user U2 in the RIS-aided full-duplex cooperative NOMA system and the cooperative NOMA system without deploying RIS are shown in Figure 3 for various values of self-interference $\gamma_{S}$ under $N=12$. Again, we can know that no matter how the power allocation coefficient and target rate are, the OP performance of the RIS-aided full-duplex cooperative NOMA system is always superior to that of the system without employing RIS. With the increasing of the residual self-interference, the OP performance gets deteriorated for both kinds of systems, meaning that the gain 
from the cooperation transmission is decreased by increasing the residual self-interference. As shown in Figure 3, if given the outage performance and the noise variance $N_{1}$, the transmission power needed by the RIS-aided cooperative NOMA system increases with the residual self-interference, which is consistent with the results of [17].

We also compare the outage performance of the near user U1 in the RIS-aided full-duplex cooperative NOMA system and the cooperative NOMA system without RIS under a series of parameters, as shown in Figures 4 and 5. For U1, the OP performance of the RIS-aided cooperative NOMA system is always superior to that of the cooperative NOMA system without RIS regardless of the power allocation coefficient and target data rate. Increasing the residual selfinterference will make U1's OP performance get degraded for these two kinds of systems. Comparing Figure 4(a) with Figure $4(\mathrm{~b})$, we know that given $R_{\text {th }}$ and transmission SNR, the OP performance of the near user gets better by increasing $w_{1}$. This is because more power is allocated to the near user. Comparing Figure 4(b) with Figure 2(b), we can know that under the same conditions, the outage performance of U1 is always inferior to that of U2 for both kinds of systems. The same conclusion can be derived through the comparison of Figure 5(b) with Figure 3(b). For example, when transmission SNR is $10 \mathrm{~dB}$, the outage performance of U1 for the RISaided system with $N=8$ in Figure 4 (b) is 0.71 while the outage performance of $\mathrm{U} 2$ for the RIS-aided system with $N=8$ in Figure 2(b) is about 0.06; the outage performance of U1 for the system without employing RIS in Figure 4(b) is around 0.99 while the outage performance of U2 for the system without employing RIS in Figure 2(b) is about 0.66. The reason for that is that the NOMA power allocation factor plays an extremely important role in improving the system performance, where the power allocation coefficient allocated to $\mathrm{U} 1$ is $w_{1}=0.4$ while to $\mathrm{U} 2$ is $w_{2}=\sqrt{1-w_{1}^{2}}=0.84$, and $w_{1}$ is lower than $w_{2}$.

\section{Conclusion}

In this paper, the OP of the RIS-aided full-duplex cooperative NOMA system is investigated. On deriving the PDF and CDF of the SNR of the reflection channel and the cooperative channel, the OP of both the near and far NOMA users is obtained. For the comparison, the OP of the conventional cooperative NOMA without RIS is also investigated. The correctness of the analytical results is verified via Monte-Carlo simulation. Simulation results show that no matter how the power allocation coefficient and target rate are, the OP performance in the RIS-aided cooperative NOMA system is always superior to that of the system without deploying RIS. With the increasing of the self-interference from fullduplex transmission, the OP performance gets deteriorated for these two systems. Power allocation has great impact on the OP performance of the RIS-aided full-duplex cooperative NOMA system, if given target rate and transmission SNR, the OP performance of the far user gets deteriorated while that of the near user gets better with increasing the power allocation coefficient to the near user. For the RIS-aided cooperative NOMA system, the OP performance improves with increasing the element number of the metasurface.

\section{Data Availability}

All information is within the paper.

\section{Conflicts of Interest}

No competing interests exist concerning this study.

\section{Acknowledgments}

This work was supported in part by the National Natural Science Foundation of China under Grant 61961007 and Grant 61862016, in part by the Guangxi Natural Science Foundation under Grant 2018GXNSFAA294093, and in part by the Training Program Funding for Thousand Young and Middle-aged Backbone Teachers of Colleges and Universities in Guangxi.

\section{References}

[1] Q. Wu and R. Zhang, "Towards smart and reconfigurable environment: intelligent reflecting surface aided wireless network," IEEE Communications Magazine, vol. 58, no. 1, pp. 106-112, 2020.

[2] W. Tang, M. Z. Chen, X. Chen et al., "Wireless communications with reconfigurable intelligent surface: path loss modeling and experimentalmeasurement," IEEE Transactions on Wireless Communications, vol. 20, no. 1, pp. 421-439, 2021.

[3] E. Basar, M. Di Renzo, J. De Rosny, M. Debbah, M.-S. Alouini, and R. Zhang, "Wireless communications through reconfigurable intelligent surfaces," IEEE Access, vol. 7, pp. 116753116773, 2019.

[4] X. Liu, Y. Liu, and Y. Chen, "Machine learning empowered trajectory and passive beamforming design in UAV-RIS wireless networks," IEEE Journal on Selected Areas in Communications, vol. 39, no. 7, pp. 2042-2055, 2021.

[5] A. R. Ndjiongue, T. Ngatched, O. A. Dobre, and H. Haas, "Towards the use of re-configurable intelligent surfaces in VLC systems: beam steering," https://arxiv.org/abs/2009 .06822 .

[6] A. Rafieifar and S. M. Razavizadeh, "Secrecy rate maximization in multi-IRS millimeter wave networks," https://arxiv.org/abs/ 2010.01113

[7] X. Mu, Y. Liu, L. Guo, J. Lin, and N. Al-Dhahir, "Capacity and optimal resource allocation for IRS-assisted multi-user communication systems," IEEE Transactions on Communications, vol. 69, no. 6, pp. 3771-3786, 2021.

[8] W. Yan, X. Yuan, Z.-Q. He, and X. Kuai, "Passive beamforming and information transfer design for reconfigurable intelligent surfaces aided multiuser MIMO systems," IEEE Journal on Selected Areas in Communications, vol. 38, no. 8, pp. 1793-1808, 2020.

[9] Z.-Q. He and X. Yuan, "Cascaded channel estimation for large intelligent metasurface assisted massive MIMO," IEEE Wireless Communications Letters, vol. 9, no. 2, pp. 210-214, 2020.

[10] X. Mu, Y. Liu, L. Guo, J. Lin, and N. Al-Dhahir, "Exploiting intelligent reflecting surfaces in NOMA networks: joint 
beamforming optimization," IEEE Transactions on Wireless Communications, vol. 19, no. 10, pp. 6884-6898, 2020.

[11] Y. Liu, X. Mu, X. Liu, M. Di Renzo, Z. Ding, and R. Schober, "Reconfigurable intelligent surface (RIS) aided multi-user networks: interplay between NOMA and RIS," https://arxiv.org/ abs/2011.13336.

[12] H. Wang, C. Liu, Z. Shi, Y. Fu, and R. Song, "On power minimization for IRS-aided downlink NOMA systems," IEEE Wireless Communications Letters, vol. 9, no. 11, pp. 18081811, 2020.

[13] Z. Ding, R. Schober, and H. V. Poor, "On the impact of phase shifting designs on IRS-NOMA," IEEE Wireless Communications Letters, vol. 9, no. 10, pp. 1596-1600, 2020.

[14] B. Zheng, Q. Wu, and R. Zhang, "Intelligent reflecting surfaceassisted multiple access with user pairing: NOMA or OMA?," IEEE Communications Letters, vol. 24, no. 4, pp. 753-757, 2020.

[15] M. Zeng, W. Hao, O. A. Dobre, and Z. Ding, "Cooperative NOMA: state of the art, key techniques, and open challenges," IEEE Network, vol. 34, no. 5, pp. 205-211, 2020.

[16] L. Zhang, J. Liu, M. Xiao, G. Wu, Y. Liang, and S. Li, "Performance analysis and optimization in downlink NOMA systems with cooperative full-duplex relaying," IEEE Journal on Selected Areas in Communications, vol. 35, no. 10, pp. 23982412, 2017.

[17] M. Elhattab, M. A. Arfaoui, C. Assi, and A. Ghrayeb, "Reconfigurable intelligent surface enabled fullduplex/ half-duplex cooperative non-orthogonal multiple access," https://arxiv .org/abs/2101.01307.

[18] X. Zhao and J. Sun, "Physical-layer security for mobile users in NOMA-enabled visible light communication networks," IEEE Access, vol. 8, pp. 205411-205423, 2020.

[19] L. Yang, X. Yan, D. B. Da Costa, T. A. Tsiftsis, H.-C. Yang, and M.-S. Alouini, "Indoor mixed dual-hop VLC/RF systems through reconfigurable intelligent surfaces," IEEE Wireless Communications Letters, vol. 9, no. 11, pp. 1995-1999, 2020.

[20] I. S. Gradshteyn and I. M. Ryzhik, Tables of Integrals, Series, and Products, Elsevier, Amsterdam, The Netherlands, 8th edition, 2015.

[21] K. P. Peppas, “Accurate closed-form approximations to generalised-K sum distributions and applications in the performance analysis of equal-gain combining receivers," IET Communications, vol. 5, no. 7, pp. 982-989, 2011. 